NSCLC

\section{Gezielte Therapie auch für Ältere}

\author{
Beim fortgeschrittenen NSCLC zählt die zielgerichtete Therapie \\ mit Erlotinib $\left(\right.$ Tarceva $^{\circledR}$ ) oder Bevacizumab (Avastin ${ }^{\circledR}$ ) zu den eta- \\ blierten Therapieoptionen. Bevacizumab ist auch im klinischen \\ Alltag sicher und effektiv.
}

Dies erläuterte Frank Griesinger, Oldenburg, anhand der Phase-IV-Studie „SAiL“. Viele der teilnehmenden Patienten wiesen Komorbiditäten auf und $74 \%$ nahmen Basismedikamente ein, jeder Dritte z.B. wegen kardiovaskulären Erkrankungen [Dansin E et al. ECCO/ESMO 2009, Abstract P9168]. ,,Trotzdem war die Kombinationstherapie mit Bevacizumab genauso gut oder sogar besser wirksam und verträglich wie in den Zulassungsstudien", betonte Griesinger. Bei $3,6 \%$ der 2.172 Patienten traten Grad-3/4-Blutungsereignisse auf, pulmonale Hämorrhagien und ZNS-Blutungen aber nur bei 0,7 bzw. 0,1 \% der Patienten. Bei 610 älteren Patienten über 65 Jahren lag die Inzidenz mit $0,7 \%$ bzw. $0,2 \%$ ähnlich niedrig [Garrido $P$ et al. ECCO/ESMO 2009, Abstract P9170].

Dabei verdoppelte sich die Ansprechrate gegenüber der herkömmlichen Chemotherapie fast, so Griesinger: $50 \%$ sprachen objektiv an, viele weitere blieben stabil, sodass eine Kontrollrate (DCR) von 88,7\% erreicht werden konnte. Bei einem medianen progressionsfreien Überleben von 7,8 Monaten in der Gesamtpopulation wie auch in der Subpopulation der Älteren und einem medianen Gesamtüberleben von jeweils 14,6 Monaten bezeichnete Griesinger das Ergebnis als fast schon dem des metastasierten kolorektalen Karzinoms vergleichbar.

Dabei werden auch ältere $\mathrm{Pa}$ tienten keinem erhöhten Risiko ausgesetzt, so Griesinger. Auch Patienten mit stabilen Hirnmetastasen könne man inzwischen die Therapie mit Bevacizumab anbieten. Ein früherer Warnhinweis bezüglich eines zunächst vermuteten erhöhten Blutungsrisikos bei ZNS-Metastasen sei inzwischen zurückgenommen worden.

Quelle: Satellitensymposium Im Rahmen des 29. Deutschen Krebskongresses am 26. Februar 2010 in Berlin; Veranstalter: Roche Pharma

Kurz notiert

Adjuvante Therapie des Mammakarzinoms: Überlebensvorteil von Letrozol gegenüber Tamoxifen

Beim krankheitsfreien Überleben von Brustkrebspatientinnen zeigen die Ergebnisse der IPCW (Inverse probability of censoring weighted)-Analyse einen Vorteil der Letrozol-Therapie gegenüber Tamoxifen von $15 \%$ (HR 0,85; $95 \%-K I 0,76-0,96)$ und $19 \%$ (HR 0,81; $95 \%-K I$ 0,69-0,96) bei der Zeit bis zur Fernmetastasierung. Zusätzlich wurde durch die Analyse eine 17\% ige Verlängerung des Gesamtüberlebens durch Letrozol gegenüber Tamoxifen gezeigt (HR 0,38; $p<0,05$ ).

In einer Hochrisikopopulation - den nodalpositiven Patientinnen - wurde ein deutlicher Vorteil für die Letrozol-Monotherapie gezeigt, verglichen mit der klassischen Sequenz (Tamoxifen gefolgt von Letrozol), so Christian Jackisch, Offenbach. Verglich man die inverse Sequenz (Letrozol gefolgt von Tamoxifen) mit Letrozol allein ergab sich dieser Unterschied laut Jackisch nicht. Nach einer neuen Auswertung der MA-17(Mammakarzinom-)Studie bietet die erweiterte adjuvante Therapie (EAT) mit Letrozol nach einer fünfjährigen Tamoxifen-Therapie einen besseren Rezidivschutz als Tamoxifen allein, so Jackisch. Beim metastasierten Mammakarzinom zeigte Jackisch anhand der Ergebnisse der ELECTRA-Studie, dass eine Kombinationstherapie aus Letrozol und Trastuzumab die Zeit bis zur Progression gegenüber der Letrozol-Monotherapie bei Patientinnen mit HER- und HR-positivem metastasiertem Mammakarzinom verlängert. Novartis Oncology

Neues Generikum bei Hirntumoren Das Temozolomid-Präparat Temomedac ${ }^{\circledR}$ ist für die Therapie von erwachsenen Patienten mit erstmalig diagnostiziertem Glioblastom begleitend zur Radiotherapie und anschließend als Monotherapie indiziert. Auch für Kinder ab drei Jahren, Jugendliche und Erwachsene mit einem nach Standardtherapie rezidivierenden oder progredienten malignen Gliom (z.B. Glioblastom oder anaplastisches Astrozytom) kann Temomedac ${ }^{\circledR}$ eingesetzt werden.

Medac

\section{Zielgerichtete Tumortherapie} Biologics mit Zwei- und Dreifachwirkung In der Forschungsabteilung von Boehringer Ingelheim wird an Biologics mit reizvollen Multifunktionen gearbeitet. Bereits heute ermöglichen Angiokinase- und Signaltransduktionsinhibitoren, apoptoseinduzierende Substanzen und monoklonale Antikörper zielgerichtete Tumortherapien. Die erzielbaren Therapiefortschritte imponieren zwar gegenüber den zuvor verfügbaren Chemotherapeutika und ermöglichen eine gewisse individualisierte Therapie, doch die Hoffnung auf Durchbrüche im Behandlungserfolg sind selten erfüllbar, erklärte Martin Schuler, Essen, im Rahmen eines Symposiums der Firma Boehringer Ingelheim anlässlich des Deutschen Krebskongresses am 25. Februar 2010 in Berlin. Boehringer Ingelheim entwickelt bereits multifunktionale Biologics:

- BIBW 2992, ein dualer, irreversibler Tyrosinkinase-Inhibitor, der für die Indikation nicht-kleinzelliges Lungenkarzinom (NSCLC), Mammakarzinom, kolorektales Karzinom sowie Kopf-Hals-Karzinome entwickelt wird. Phase-IIStudien mit BIBW 2992 zeigen hohe Ansprechraten, partielle Remissionen sowie eine sehr gute Krankheitskontrolle, so Schuler.

- BIBF 1120 (voraussichtlich Vargatef' ${ }^{\mathrm{TM}}$ ) als dreifach wirksamer oraler Angiokinase-Hemmer, der sich in der klinischen Erprobung für die Indikation NSCLC sowie Ovarialkarzinom befindet. Wie Alexander Schmittel, Berlin, auseinandersetzte, könnte die Substanz auch beim kolorektalen Karzinom sowie beim Nierenzellkarzinom wirksam sein. Die ersten Phase-II-Studienergebnisse seien vielversprechend, so Schmittel.

Zur Zeit läuft die Patienten-Rekrutierung für die Phase-III-Studie "LUME-Lung" mit BIBF 1120 plus Standard-Chemotherapie beim fortgeschrittenen NSCLC in der Zweitlinientherapie. TUK 